9 Kelleher I, Harley M, Murtagh A, Cannon M. Are screening instruments valid for psychotic-like experiences? A validation study of screening questions for psychotic-type experiences using in-depth clinical interview. Schizophr Bull 2009; 19 June (Epub ehead of print)

10 Nesse RM. Evolution at 150: time for truly biological psychiatry. Br J Psychiatry 2009; 195: 471-2.

11 Crow TJ. Is schizophrenia the price that Homo sapiens pays for language? Schizophr Res 1997; 28: 127-41.

12 Burns JKP. An evolutionary theory of schizophrenia: cortical connectivity, metarepresentation and the social brain. Behav Brain Sci 2004; 27: 831-85.

13 Nesse RM. Cliff-edged fitness functions and the persistence of schizophrenia (commentary). Behav Brain Sci 2004; 27: 862-3.

14 Dodgson G, Gordon S. Avoiding false negatives: are some auditory hallucinations an evolved design flaw? Behav Cogn Psychother 2009; 37 325-34
15 van OS J. A salience dysregulation syndrome. Br J Psychiatry 2009; 194: 101-3.

16 Craddock N, Owen MJ. The Kraepelinian dichotomy - going, going . . . but still not gone. Br J Psychiatry 2010; 196: 92-5.

17 Jenner JA. HIT: Hallucination focused integrative therapy. In Hallucinations: A Practical Guide (eds F Laroi, A Aleman): 163-82. Oxford University Press, 2010.

18 Andrews G, Poulton R, Skoog I. Lifetime risk for depression: restricted to a minority or waiting for most? Br J Psychiatry 2005; 187: 495-6.

19 Moffitt TE, Caspi A, Taylor A, Kokaua J, Milne BJ, Polanczyk G, et al. How common are common mental disorders? Evidence that lifetime prevalence rates are doubled by prospective versus retrospective ascertainment. Psychol Med 2010; 40: 899-909.

20 Dobzhansky T. Nothing in biology makes sense except in the light of evolution. American Biol Teach 1973; 35: 125-9.

\section{psychiatry in pictures}

\title{
Bipolar (2008)
}

Alice Hatter (b. 1979)

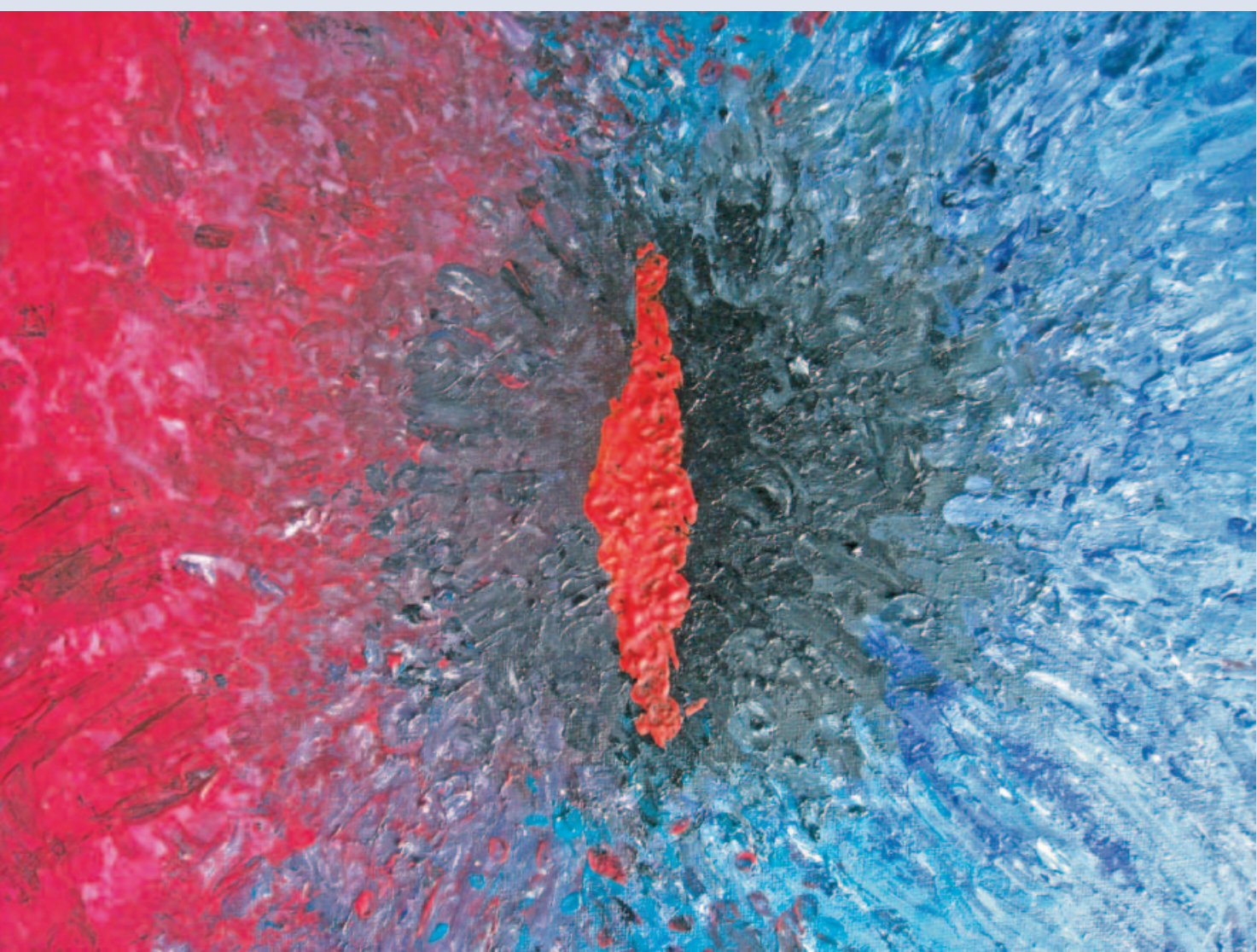

Alice Hatter decided to leave her medical studies after developing a bipolar illness. This decision was not made because she felt that her illness prevented her from continuing or coping with her studies, but because her outlook on life changed afterwards. Being able to express herself and her experiences through the medium of art has been a great help to her while trying to combat her illness. She had always enjoyed the arts when growing up and hopes to develop this area of her life further. Alice's interest lies in the link between creativity and mental illness, in particular affective disorders, and she hopes in the future to have the opportunity to look more closely at this area of mental health.

This picture represents Alice's idea and experience of a bipolar illness. One side is blue and the other is pink, representing depression and mania, respectively. The colours get deeper as you go to the centre where the core appears black - but it is actually black on the depression side and very dark purple on the mania side. The centre is open to interpretation - the red can either represent 'rage' or 'anger' which is at the centre of the illness but gets buried under the other emotions; or it could be the rawness of the person when the 'end point' of mania or depression is reached; it can be like falling into the depths and being exposed and naked. Around the top and bottom edges of the picture the colours merge into one another to give a turquoise colour eventually which Alice feels represents the 'normal' mood state, but the fact that all the colours merge like a spectrum is still preserved as, like the illness, it is a continuum rather than specific discrete entities.

Edited by Allan Beveridge. 